\title{
Orientation of Crystallites in Natural Rubber under different Modes of Load
}

\author{
K. Schneider \\ Leibniz-Institut für Polymerforschung Dresden, Germany \\ S. Roth \\ DESY, Hamburg, Germany
}

\begin{abstract}
Strain induced crystallization (SIC) is one of the .most influencing factors for the outstanding mechanical properties of natural rubber. It was extensively investigated within the last decades (e.g. Zhang et al. 2013, Brüning 2014). In practical use, and mainly in the case of pre-cracked respectively damaged samples, the strain field around the crack tip is highly inhomogeneous. This will influence the strain induced crystallization. Therefore, the effective crystallite distribution and orientation around the crack tip will be as well inhomogeneous. In contrast to the uniaxial tensile load of rubber with fibre symmetrical distribution of crystallites, in pure shear samples no fibre symmetry is found. The crystallite orientation with respect to the sample geometry will be investigated via x-ray scattering. and the results are used to describe the crystallization under inhomogeneous stretching conditions.
\end{abstract}

\section{INTRODUCTION}

Synchrotron X-ray scattering in the small and wide angle range (SAXS and WAXS) enables online investigation of structural changes during deformation. By WAXS crystallization processes can be followed and the orientation or orientation distribution of crystallites can be estimated. During steady loading only one pattern per deformation can be picked up.

For the evaluation of scattering data of samples under uniaxial load mainly presence of fibre symmetry is assumed. This means, the tensile direction is the fibre axis, around which the structural features, e.g. crystallites, are homogeneous distributed without any preferred direction. So it is sufficient for the evaluation to take one pattern and to assume that the pattern of the sample rotated around the fibre axis doesn't change.

In the case of some constraints in the sample, e.g. by the sample geometry, it is necessary to check, whether the assumption of fibre symmetry is fulfilled.

In the present presentation it will be investigated, whether for pure shear geometry fibre symmetry is fulfilled and which information can be obtained by sample rotation within the beam.

\section{EXPERIMENTAL}

\subsection{Sample preparation and deformation}

Samples of natural rubber, unfilled and filled with 20 phr carbon black (NR0 and NR20), were investi- gated. The samples have pure-shear geometry with the dimensions of $80 \mathrm{~mm}$ (width) $* 10 \mathrm{~mm}$ (hights) * $1 \mathrm{~mm}$ (thickness), the bulge on both sides has a diameter of $5 \mathrm{~mm}$, it enables a defined clamping of the samples. On the free sample length 2 lines were applied for optical strain estimation.

The samples were externally quasi-statically stretched to a stretch ratio of 5 and 6 , respectively, (NR0_5, NR0_6 and NR20_5) and fixed within a frame. With this frame they were put into the beam.

\subsection{Synchrotron WAXS measurements}

The synchrotron WAXS measurements were performed at DESY, Hamburg, beamline P03 with a micro beam, a wavelength of $0.094 \mathrm{~nm}$ (energy of $13.2 \mathrm{keV}$ ) and a beam size of about $20 \mu \mathrm{m} * 20 \mu \mathrm{m}$ were used. The general arrangement of the measurements is shown in Fig. 1.

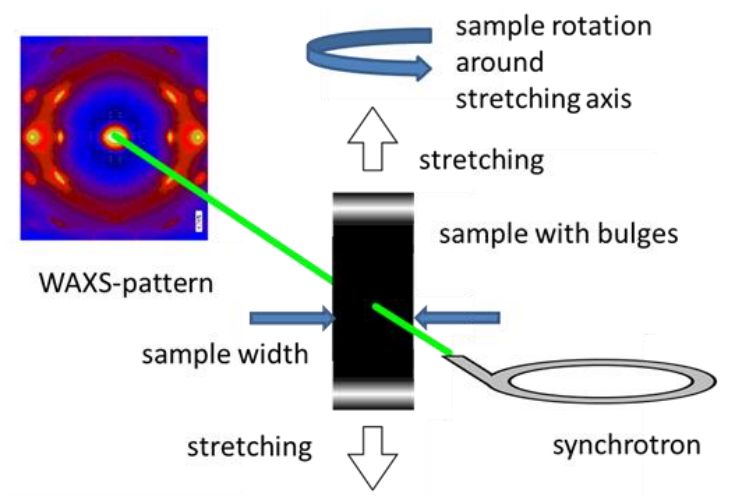

Figure 1. Arrangement of the sample in the beam of the synchrotron source. The equatorial reflexes have the indices

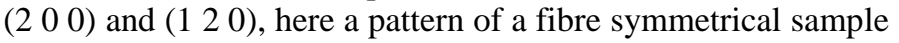
is shown. 
For the measurement a PILATUS 300k-detector was used with a detector distance of $206 \mathrm{~mm}$. The intensity of the incoming beam was measured with an ionisation chamber, the intensity of the transmitted beam by a beamstop diode.

While for samples with small width the assumption of fibre symmetry is valid, here samples with high width with respect to the length were investigated. The samples were investigated at 7 different rotation angles of the sample with respect to the beam from $-45^{\circ}$ to $45^{\circ}$ in steps of 15 degree. At each arrangement 10 patterns were captured scanning along the sample in stretching direction with a step width of $1 \mathrm{~mm}$ with an exposure time of $1 \mathrm{~s}$.

\subsection{Primary data processing}

For the samples absorption coefficients $\mu$ of 0.418 and $0.44 \mathrm{~mm}^{-1}$ (NR0 and NR20) were estimated. The raw patterns were corrected with respect to the beam intensity, the background scattering and the sample thickness, which depends from the stretch ratio as well as from the tilting angle.

Due to the tilting of the sample the x-ray path within the sample is changed, see Fig. 2.

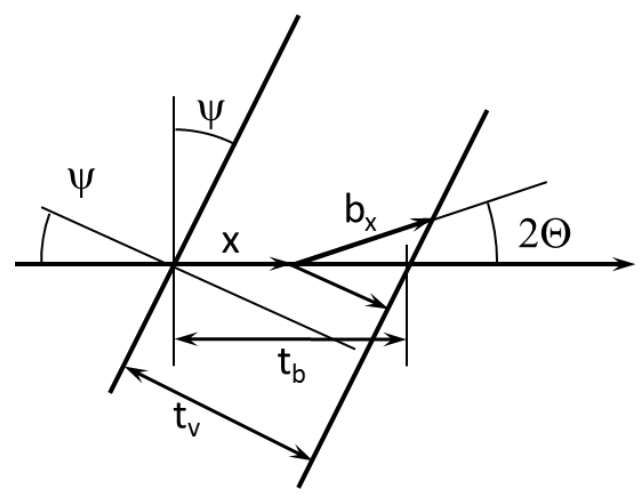

Figure 2. Sketch for the correction of different beam paths within the sample due to tilted sample orientation, tilting angle $\psi$ between incoming beam and sample. Instead of the initial thickness $t_{v}$ of the sample the beam passes the thickness $t_{b}$, while the path of the scattered beam is $x+b_{x}$.

The intensity of the scattered beam changes according due to this rotation via the angle $\psi$ to

$S(2 \theta)=\frac{I_{t_{b}}}{I_{0} F t_{b}} \exp \left(\frac{\mu t_{b} \cos (\psi)}{\cos (\psi+2 \theta)}\right)$

The patterns, corrected by this way, are shown in Fig. 3.

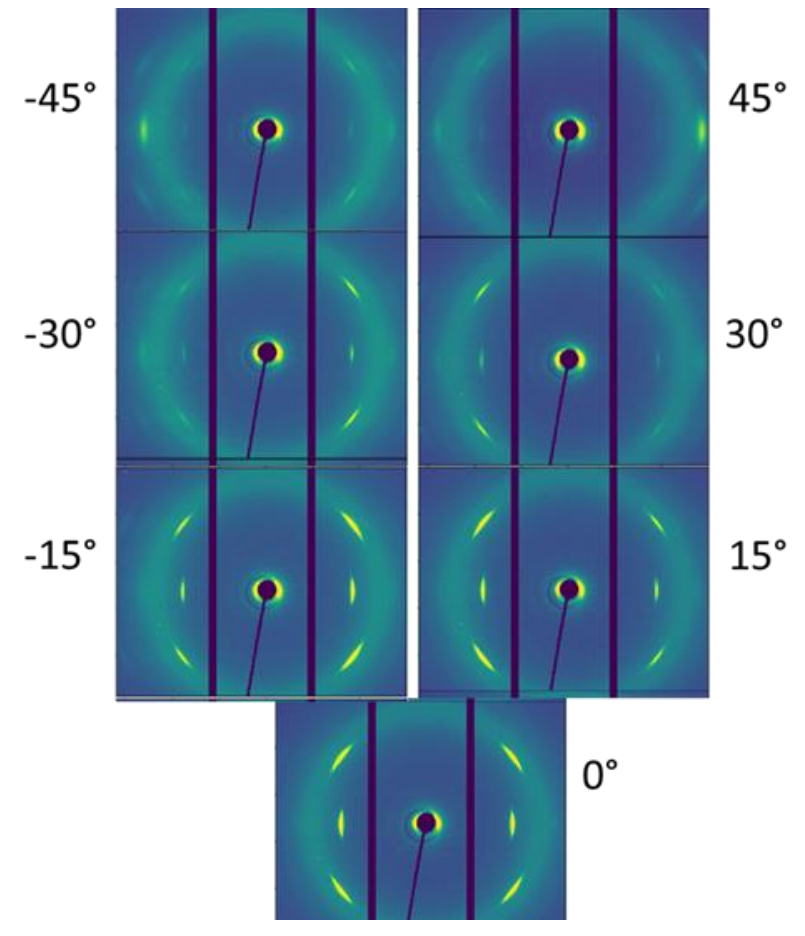

Figure 3. Patterns of the sample NR0_5 after intensity-, thickness- and tilt-correction in dependence of the angle of rotation of the sample around the tensile axis. Rotation angle $\pm 45^{\circ}$, $\pm 30^{\circ}, \pm 15^{\circ}, 0^{\circ}$ (from top to bottom).

It is obvious, that there is a very strong dependence of the individual peak intensities dependent on the rotation angle of the sample, what confirms that there is no fibre symmetry within the samples.

Generally it is obvious, that the c-axis of the crystallites is strongly oriented with respect to tensile axis. The further discussion is focused on the equatorial peaks $\left(\begin{array}{lll}2 & 0 & 0\end{array}\right)$ and $\left(\begin{array}{lll}1 & 2 & 0\end{array}\right)$.

The characterization of the crystalline peaks was done via integrating the peak area over the amorphous halo.

\section{ESTIMATION OF CRYSTALLITE ORIENTATION FUNCTION}

\subsection{Crystallographic characterization of strain- induced crystallites in natural rubber}

The crystal structure of natural rubber was intensively investigated in the past. Takahashi \& Kumano (2004) gives a comprehensive overview about these investigations. The unit cell is monoclinic with parameters $\mathrm{a}=12.41 \AA, \mathrm{b}=8.81 \AA$ and c (fibre axis) = $8.23 \AA$, and $\beta=93.1^{\circ}$. The structure factors of the

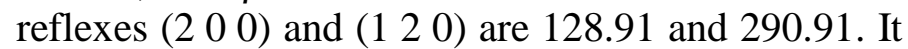
is very similar to the orthorhombic cell, reported by Nyburg (1954), which should be used for reasons of simplicity for the further evaluation. 


\subsection{Peak intensities and crystallite orientation}

The characteristic peaks of a pattern appear if the Bragg condition is fulfilled. So we get for each of the left and right equatorial peak (2 00 ) one possible orientation of crystallites, for each of the (1 20$)$ peaks two possible orientations of the scattering crystallites.

We assume a random orientation of crystallites superimposed with a Gaussian distribution of oriented crystallites according to

$X_{1}(\varphi)=C_{0}+C_{1} \exp \left(-\frac{(\varphi)^{2}}{2 \sigma^{2}}\right)$

with the intensity-constants $C_{0}$ and $C_{1}$, the variance $\sigma^{2}$ over the orientation angle $\varphi$. The details of scattering geometry are shown in Fig. 4.

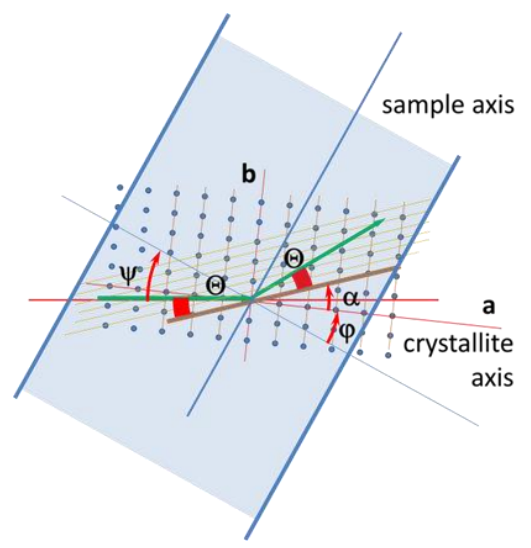

Figure 4. Detailed scattering geometry (here for the reflex (1 20 ): The sample is rotated by an angle $\psi$ to the beam, the aaxis of an individual crystallite has an angle of $\varphi$ within the sample, the scattering crystalline plane has an angle of $\alpha$ to the a-axis. The scattering angle $\theta$ is between the incoming beam and the scattering plane as well as between this and the scattered beam.

This distribution enables the description of the peak intensities in a quite satisfying way, see Fig. 5.

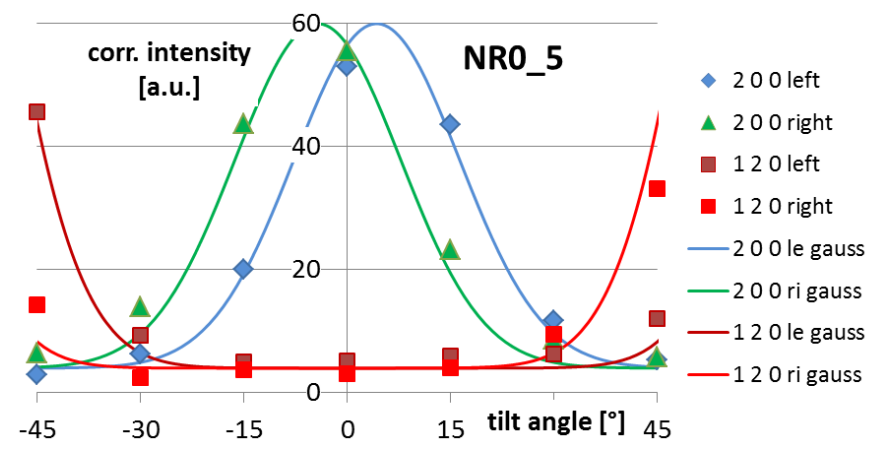

Figure 4. Measured intensities of the crystallite peaks and model of the intensities using a Gaussian distribution for the orientation distribution of crystallites in the unfilled stretched natural rubber, stretch ratio 5 .

It is obvious, that nearly no random distributed crystallites are found.

\subsection{Influence of strain on the crystallite orientation function}

With increasing of the stretch ratio, here from 5 to 6 for NR0, the scattering intensities over the rotation angle change slightly, see Fig. 5.

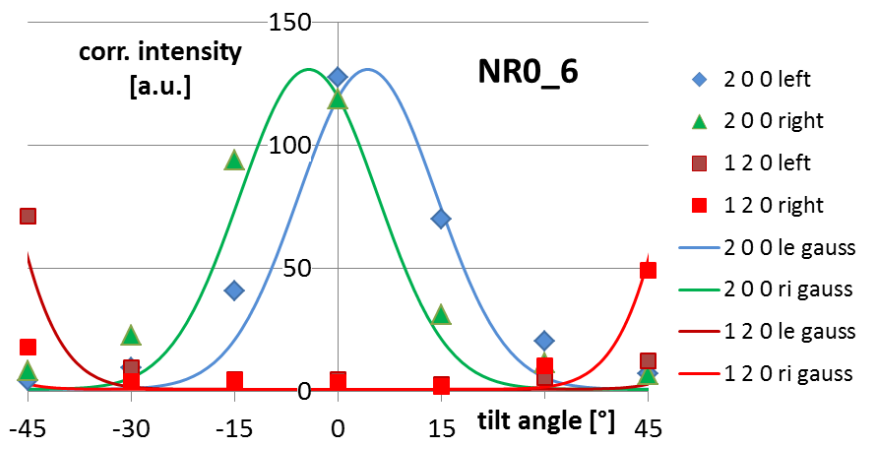

Figure 5. Intensities of the crystalline peaks of the sample NR0 with a stretch ratio of 6 in dependence of sample rotation, measured values and values of the model.

Mainly the orientation distribution becomes narrower.

\subsection{Influence of filler content on the crystallite orientation}

In the case of filled NR the superstructure is remarkable different. The general orientation of the crystallites is similar, but an enormous amount of random distributed crystallites appears, see Fig. 6.

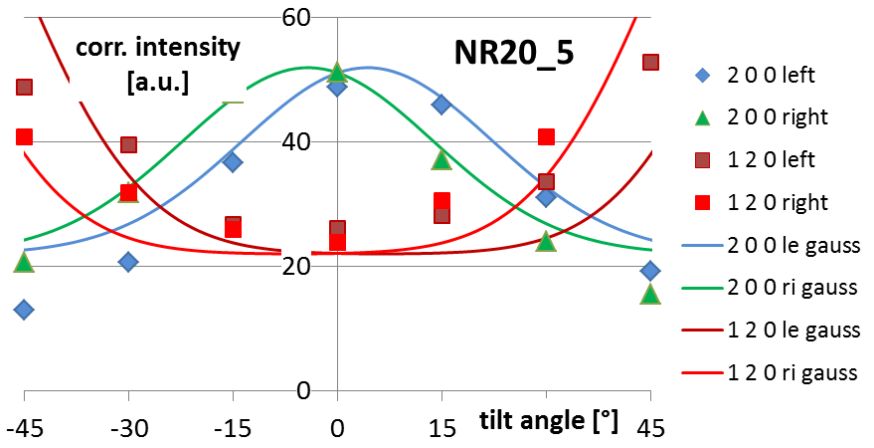

Figure 6. Intensities of the crystalline peaks of the sample NR20 with a stretch ratio of 5 in dependence of sample rotation, measured values and values of the model.

The parameters, describing the orientation distribution of the crystallites are summarized in Table 1 .

Table 1. Parameter of the orientation distribution of the a-axis of crystallites.

\begin{tabular}{lccc}
\hline reflex & $\mathrm{C}_{0}$ & $\mathrm{C}_{1}$ & $\sigma^{2}$ \\
\hline NR0_5 & 2 & 28 & 144 \\
NR0_6 & .5 & 65 & 100 \\
NR20_5 & 11 & 15 & 324 \\
\hline
\end{tabular}




\subsection{Crystallite orientation in tensile direction}

The width of orientation distribution of the crystallites in stretching direction can be estimated by the width of the equatorial reflexes in azimuthal direction. In Table 2 the values are summarized.

Table 2. Width of the orientation distribution (deviation from stretching direction).

\begin{tabular}{lcc}
\hline reflex & 200 & 120 \\
\hline NR0_5 & 10 & 12 \\
NR0_6 & 11 & 11.6 \\
NR20_5 & 16 & 16 \\
\hline
\end{tabular}

\subsection{Crystallite size, estimated by Scherrer formula}

The Scherrer formula enables the rough estimation of crystallite sizes. It is

$L_{h k l}=\frac{B \lambda}{\Delta(2 \theta) \cos \left(\theta_{0}\right)}$

where $L_{h k l}$ is the size of the crystallite perpendicular to the lattice plane ( $h k l$ ), $\lambda$ the wavelength of the used radiation, $\Delta(2 \Theta)$ total half width of the reflex, $\Theta_{0}$ the Bragg angle in radian and $K$ the ScherrerFormfactor in the order of 1 .

The mean sizes of the crystallites in different directions are summarized in Table 3.

Table 3. Size of crystallites, estimated by the Scherrer Formula.

\begin{tabular}{lcc}
\hline reflex & 200 & 120 \\
\hline NR0_5 & 25 & 8.8 \\
NR0_6 & 26.1 & 8.9 \\
NR20_5 & 19.6 & 7.6 \\
\hline
\end{tabular}

\section{DISCUSSION}

Comparing the 10 patterns at each strain/rotation angle show no significant difference. This confirms that the crystalline structure within the sample is quite homogeneous at a scale of microns.

Investigating a stretched pure shear sample under different angles shows a strong dependence of the individual crystalline reflexes at the same strain. This is a clear indication, that there is no fibre symmetry.

The evaluation of the different peaks enables the estimation of the orientation of the crystallites. It can be described by a Gaussian distribution. Here it is found, that the a-axis of the crystallites is mainly oriented perpendicular to the sample surface.

For unfilled natural rubber there are no random distributed crystallites found. With increasing strain the orientation of the crystallites becomes slightly narrower, while the size of the individual crystallites remains mainly constant.

In the case of CB-filled natural rubber the establishment of strain induced crystallization is remarkably influenced by the presence of filler. The sizes of crystallites are, compared with the unfilled rubber, generally smaller, and the orientation of crystallites becomes wider. Furthermore, additional to the oriented crystallites we found a remarkable amount of randomly distributed crystallites. Obviously, this effect is also caused by the constraint of crystallization due to the spaces between the filler particles, and not only by the constraining sample geometry.

\section{CONCLUSIONS}

The investigations show, that a constraining geometry as well as fillers have an impact on the orientation of strain induced crystallites in natural rubber. Due to the reinforcing effect of the crystallites this will therefore have an impact not only on the mechanical behaviour of anisotropic loaded rubber material, but also on their crack growth resistance. The anisotropic load ahead of the crack tip will influence the orientation of crystallites in the vicinity of the crack tip and, furthermore, the details of the failure process.

These details will be the topic of further investigations.

\section{ACKNOWLEDGEMENT}

J. Domurath from IPF, Dresden, and $\mathrm{M}$. Schwarzkopf \& A. Rothkirch from DESY, Hamburg, are acknowledged for their support performing the WAXS experiments and evaluating the data. Furthermore we thank SRI for financial support.

\section{REFERENCES}

Zhang, H., A. K. Scholz, Y. Merckel, M. Brieu, D. Berghezan, E. J. Kramer and C. Creton 2013. Strain induced nanocavitation and crystallization in natural rubber probed by real time small and wide angle X-ray scattering. Journal of Polymer Science Part B-Polymer Physics 51(15), 1125-1138.

Brüning, K. 2014. In-situ Structure Characterization of Elastomers during Deformation and Fracture. Cham, Heidelberg, New York, Dordrecht, London: Springer

Takahashi, Y. \& Kumano, T. 2004. Crystal Structure of Natural Rubber. Macromolecules 37, 4860-4864.

Nyburg, S. C. 1954. A Statistical Structure for Crystalline Rubber. Acta Crystallographica 7, 385-392. 